\title{
El género Panphalea (Compositae: Nassauvieae) en Uruguay
}

\author{
CRISTINA TRUJILLO ${ }^{1}$, LILIANA KATINAS ${ }^{2}$ y JOSE M. BONIFACINO ${ }^{1}$
}

\begin{abstract}
Resumen: Se realiza la revisión taxonómica de Panphalea Lag. (Compositae: Nassauvieae) para Uruguay. Panphalea en Uruguay consiste de cinco especies: Panphalea bupleurifolia Less., Panphalea cardaminifolia Less., Panphalea commersonii Cass., Panphalea heterophylla Less. y Panphalea maxima Less. Todas las especies uruguayas fueron evaluadas y circunscriptas en base al análisis de los protólogos, análisis de laboratorio, estudio cuidadoso del material tipo, observación de especímenes de herbario y observaciones a campo. Se presenta una clave dicotómica para la identificación de las especies reconocidas para el Uruguay. Para cada especie se provee sinonimia, descripciones ampliadas, distribución geográfica y dibujos. Se designan dos neotipos.
\end{abstract}

Palabras clave: Asteraceae, conservación, Mutisioideae, Nassauvieae, taxonomía.

Summary: The genus Panphalea (Compositae: Nassauvieae) in Uruguay. A taxonomic revision of Panphalea Lag. (Compositae: Nassauvieae) for Uruguay is presented. Panphalea, in Uruguay, is composed of five species: Panphalea bupleurifolia Less., Panphalea cardaminifolia Less., Panphalea commersonii Cass., Panphalea heterophylla Less., and Panphalea maxima Less. All uruguayan species of Panphalea were taxonomically evaluated and circumscribed based on analyses of the protologues, laboratory studies, and careful study of type material, herbarium specimens and field observations. A dichotomous key for the identification of the Uruguayan species is provided. For each species, synonymy, detailed description, geographic distribution, and line drawings are presented. Two neotypes are designated.

Key words: Asteraceae, conservation, Mutisioideae, Nassauvieae, taxonomy.

\section{INTRODUCCIÓN}

En Uruguay la familia Compositae se compone de 374 especies entre nativas y adventicias y constituye la segunda familia de plantas vasculares en número de especies (Marchesi, com. pers.). Dentro de la familia Compositae, la tribu Nassauvieae en Uruguay se compone de 18

\footnotetext{
1 Laboratorio de Botánica, Departamento de Biología Vegetal, Facultad de Agronomía, Universidad de la República, Casilla de Correo 1238, Montevideo, Uruguay. Email: cristgunino@gmail.com, bonifacinoj@ fagro.edu.uy.

2 División Plantas Vasculares, Museo de La Plata, Paseo del Bosque s/n, 1900 La Plata, Argentina. Email: katinas@fcnym.unlp.edu.ar.
}

especies reunidas en seis géneros (Katinas, 2008): Criscia Katinas (1 especie), Holocheilus Cass. (3 especies), Jungia L. f. (1 especie), Panphalea Lag. (5 especies), Perezia Lag. (3 especies) y Trixis P. Browne (5 especies). Los géneros de la tribu Nassauvieae se diferencian del resto de las Compositae por sus corolas bilabiadas y sus estilos de ramas truncadas provistas de una coronita de papilas apicales (Fig. 1) (Crisci, 1974; Katinas et al., 2008).

Panphalea es un género compuesto de nueve especies, las que se distribuyen en el sur de Brasil, Uruguay, este de Paraguay y nordeste de Argentina (Cabrera, 1953, 1959). El principal centro de diversidad de Panphalea es el estado de Rio Grande do Sul (Brasil), donde se encuentran todas las especies descriptas para el 
Bol. Soc. Argent. Bot. 49 (1) 2014

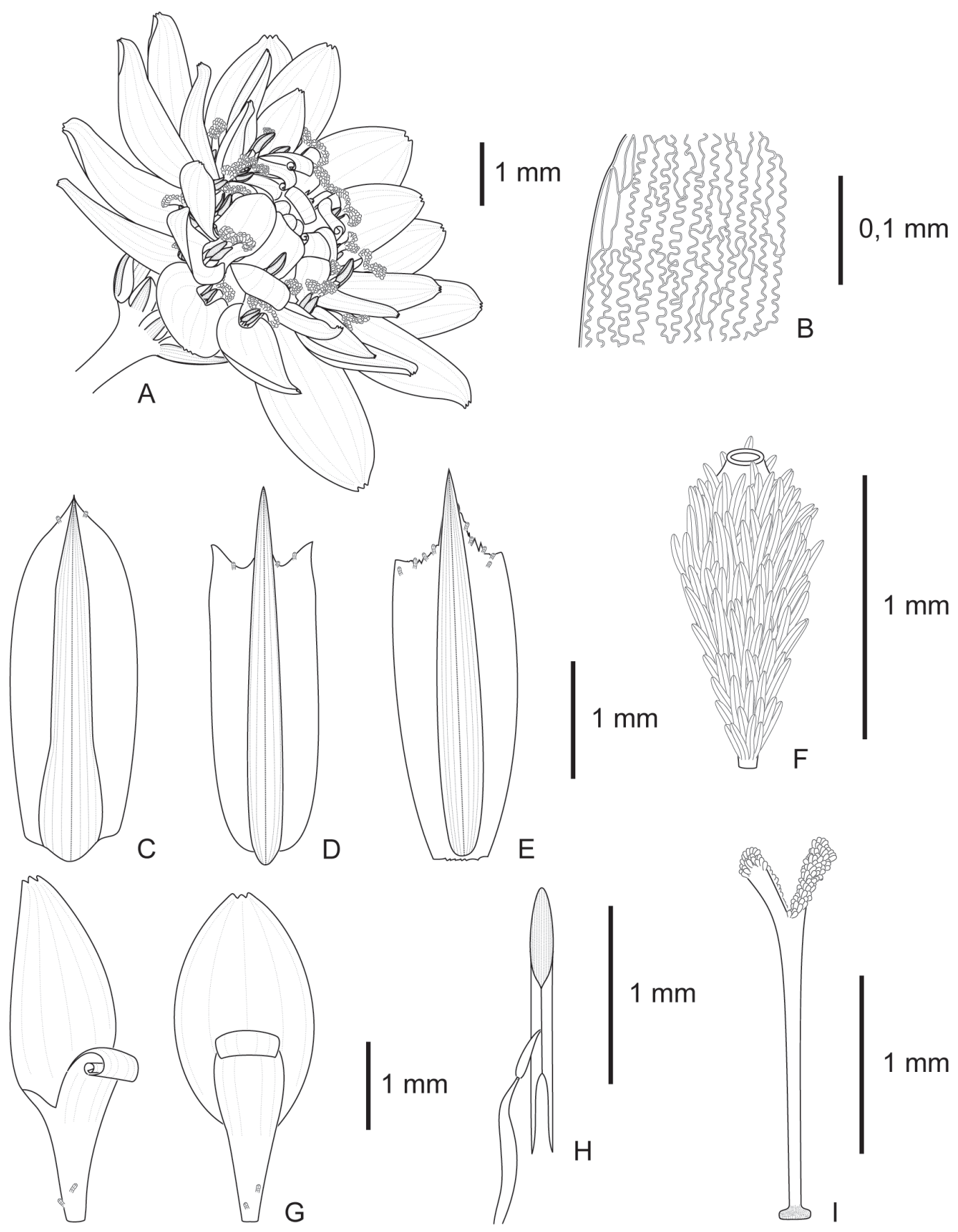

Fig. 1. Aspectos morfológicos de Panphalea. A: Capítulo de Panphalea bupleurifolia. B: Superficie de la filaria en Panphalea maxima vista al microscopio. C: Filaria del involucro de Panphalea bupleurifolia. D: Filaria del involucro de Panphalea commersonii. E: Filaria del involucro de Panphalea maxima. F: Cipsela en Panphalea commersonii. G: Corolas (vista lateral y frontal) en Panphalea bupleurifolia. H: Estambre en Panphalea bupleurifolia. I: Estilo y estigma en Panphalea bupleurifolia. (A, C, G: Lema s.n., MVFA 6737; B, E: Olano et al. s.n., MVFA 8768; D, F: Berro 683, MVFA; H, I: Bayce \& Marchesi s.n., MVFA 19956). 
género (Mondin \& Baptista, 1996; Katinas \& Crisci, 2008). Más recientemente, Monge (2010) cita para Brasil 5 especies (P. araucariophila Cabrera, P. cardaminifolia Less., P. maxima Less., P. ramboi Cabrera y P. smithii Cabrera). Esta actualización marca un claro contraste con lo anteriormente reportado ( 9 especies) lo cual resulta particularmente llamativo, ya que entre las cuatro especies faltantes hay dos especies muy comunes al norte de Uruguay ( $P$. heterophylla Less. y $P$. commersonii Cass.) las cuales habitan ambientes que se extienden al sur de Brasil. La revisión del género, actualmente en curso por uno de los autores (CT) confirmará estos datos.

Interesantemente, de las cinco especies de Panphalea citadas para Uruguay, cuatro de ellas fueron descriptas sobra la base de materiales colectados en el país: Panphalea cardaminifolia, P. commersonii, $P$. heterophylla, y P. maxima. Panphalea bupleurifolia Less es la única de las cinco especies citadas para Uruguay descripta a partir de material proveniente de Brasil.

Gibert (1873), en el primer catálogo de plantas vasculares para Uruguay, cita cuatro especies: Panphalea bupleurifolia, P. commersonii, P. heterophylla y P. tweedii Gard. \& Field. Arechavaleta (1906) no considera a Panphalea tweedii e incorpora a $P$. cardaminifolia y $P$. maxima a la lista de especies presentes en el país. Herter (1930) cita para Uruguay seis especies, al añadir $P$. tweedii al listado presentado anteriormente por Arechavaleta (1906). En su revisión del género Panphalea, Cabrera (1953) incluye a $P$. tweedii en la sinonimia de $P$. heterophylla. Finalmente, Katinas (2008) mantiene la lista de cinco especies presentadas por Arechavaleta (1906): P. bupleurifolia, P. cardaminifolia, $P$. commersonii, $P$. heterophylla y $P$. maxima. Katinas y Crisci (2008) realizaron un análisis cladístico y biogeográfico del género y establecieron a la región Paranense, en Brasil, como su posible área de origen. Estos autores sugirieron además que, dada la asociación frecuente de Panphalea a márgenes de ríos y arroyos, la dispersión hidrófila de sus especies es altamente probable.

Cabrera (1953) en su revisión del género realizó breves descripciones de las especies, enfatizando los caracteres foliares. En el presente trabajo se amplían las descripciones de las especies uruguayas, incluyendo además los caracteres reproductivos. Asimismo, se amplían las citas geográficas de las especies en el Uruguay, se analiza la ecología de las mismas y se asignan categorías del estado de conservación.

\section{Materiales y Métodos}

Se consultaron especímenes depositados en los herbarios LP, MVFA, MVJB y MVM. Paralelamente se realizaron observaciones de material fresco en los ambientes donde las especies ocurren naturalmente. Se consideraron 62 caracteres morfológicos, vegetativos y reproductivos. Estos caracteres fueron observados en cada espécimen, registrándose los datos obtenidos en tablas (una por cada espécimen), a partir del análisis de las cuales se obtuvo la descripción del género y las especies, e información para la elaboración de la clave de identificación de las mismas. Para la morfología de tricomas se siguió la terminología propuesta por Ramayya (1962).

Las mediciones se realizaron con un calibre y una regla de precisión $1 / 20 \mathrm{~mm}$ y $1 / 2 \mathrm{~mm}$, respectivamente. Se hicieron observaciones por medio de una lupa binocular. Las dimensiones del involucro y las longitudes de filarias y cipselas fueron realizadas en material seco, mientras que el resto de los demás caracteres utilizados fueron medidos en estado hidratado. Para la observación de tricomas se realizaron preparados no permanentes los cuales fueron estudiados bajo el microscopio óptico.

Los dibujos de las distintas partes florales se realizaron con cámara clara anexada a la lupa estereoscópica y al microscopio. A partir de las etiquetas de herbario se registraron datos fenológicos, ambientes ocupados y distribución geográfica, los cuales fueron complementados a partir de la literatura existente y observaciones de campo.

Se evaluaron las especies a nivel país, según las categorías y criterios de la lista roja de la UICN: versión 3.1 (IUCN, 2012), las directrices para el uso de la misma, versión 10.1 (IUCN, 2013), y las directrices para emplear los criterios a nivel regional y nacional: versión 4.0 (UICN, 2012); se asignó para cada especie una categoría de conservación. 
Bol. Soc. Argent. Bot. 49 (1) 2014

\section{Tratamiento TAXonómico}

Clave para la identificación de los géneros de Nassauvieae presentes en Uruguay.

1. Papus ausente

Panphalea

1'. Papus presente

2. Receptáculos paleáceos Jungia

2'. Receptáculos desnudos

3. Corolas enteramente amarillas o anaranjadas

4. Plantas herbáceas, roseta basal presente, hojas adpresas al suelo, elípticas a orbiculares, ápice obtuso; plantas escaposas, capítulos solitarios o en capitulescencias paucicéfalas de dos, o raramente tres capítulos

Criscia

4'. Plantas subarbustivas o arbustivas, roseta basal generalmente ausente, si presente entonces hojas ascendentes, elípticas; plantas no escaposas, capítulos numerosos (más de 10) por capitulescencia

Trixis

3'. Corolas blancas o violáceas, a veces con el labio interior amarillo

5. Hojas de margen sinuoso o lobulado, lóbulos redondeados. Cipselas papilosas, contraídas a rostradas en el ápice

Holocheilus

5'. Hojas de margen generalmente dentado o aserrado, dientes agudos, provistos generalmente de tricomas rígidos. Cipselas truncadas en el ápice, velludas Perezia

Panphalea Lag. Amen. Nat. Españ. 1: 34. 1811. Tipo: Panphalea commersonii Cass. Fig.1.

Hierbas anuales o perennes con rizoma o con xilopodio; hojas inferiores en roseta, enteras o divididas, pecioladas o sésiles, amplexicaules o semiamplexicaules, venación pinnatinervada o palmatinervada; hojas superiores alternas, sésiles, amplexicaules o semiamplexicaules; capitulescencias corimbiformes o paniculiformes, ocasionalmente capítulos solitarios en las plantas pequeñas, capítulos pedicelados; bractéolas enteras, ápice agudo, escariosas; involucro acampanado o cilíndrico; filarias dispuestas en una o dos series subiguales, rígidas, con el margen anchamente escarioso; receptáculo plano, alveolado o tuberculado, glabro, desnudo; flores isomorfas, hermafroditas, corolas blancas, bilabiadas, con tricomas glandulares escasos o ausentes (biseriados vesiculares subtipo beta), labio abaxial blanco, 3-dentado, labio adaxial blanco o amarillo, 2-dentado; anteras sagitadas, con apéndice conectival elíptico u ovado, agudo; estilo bífido, de ramas truncadas, provistas de corona apical de papilas colectoras; cipselas obovoides, elipsoides, pubescentes, con tricomas no glandulares gemelos, costillas 4 a 6, carpopodio simétrico; papus ausente.

De acuerdo con Katinas (1995) y Katinas et al. (2008), la razón del cambio ortográfico a "Pamphalea" propuesta por de Candolle (1812) no es justificada, debiendo utilizarse la forma original (Panphalea) según fuera descripta por Lagasca (1811).

Clave para la identificación de las especies de Panphalea Lag. en Uruguay.

1. Hojas basales angostamente ovadas a lineares, enteras. Panphalea bupleurifolia

$1^{\prime}$. Hojas basales orbiculares o lirado-pinnadas

2. Tallos provistos de base tuberosa (xilopodio). Hojas con pecíolos cilíndricos

Panphalea commersonii

$2^{\prime}$. Tallos sin base tuberosa. Hojas con pecíolos planos. 
3. Plantas pequeñas, generalmente menores a $20(-23) \mathrm{cm}$ de altura; capitulescencias corimbiformes; labio adaxial de las corolas amarillo Panphalea heterophylla

$3^{\prime}$. Plantas grandes, generalmente mayores a $20 \mathrm{~cm}$ de altura; capitulescencias paniculiformes; labio adaxial de las corolas blanco

4. Hojas basales lirado-pinnatisectas a pinnatipartidas, lóbulos entero-dentados

Panphalea cardaminifolia

4'. Hojas basales lirado-pinnatífidas, lóbulos crenados-dentado

..Panphalea maxima

Panphalea bupleurifolia Less. Linnaea 5: 8 . 1830. Tipo: Brasil. Brasilia meridionali, Sellow s.n. (Holotypus B, destruido, foto F16094!) Neotypus aquí designado: Uruguay. Florida, Estancia Santa Clara, Timote, 9-X-1943, Gallinal et al. PE-5294 (MVFA!). Duplicado del neotypus: MVM 8680! Fig. 2A.

Hierbas perennes, con rizoma grueso, 25-115 $\mathrm{cm}$ de altura, tallos erectos, estriados, tricomas glandulares de ausentes a escasos; hojas inferiores 5-29 x 0,1-1,1 cm, muy numerosas, pecíolo no definido, plano, envainador, lámina angostamente obovada a linear, base atenuada, ápice agudo a obtuso, margen entero, tricomas glandulares de ausentes a escasos; hojas superiores 1-18,6 x 0,1-0,9 cm, amplexicaules, ovadas, largamente atenuadas hacia el ápice, base cordada, ápice agudo, margen entero, tricomas glandulares de ausentes a escasos; capitulescencias corimbiformes, capítulos $5-75$, pedicelos $0,2-5,8 \mathrm{~cm}$ de longitud; bractéolas $1-5,0,8-2 \times 0,2-1 \mathrm{~mm}$, ovadas; involucro 3-4,5 x 2,9-4,5 mm, acampanado o cilíndrico; filarias 9 a 14 , dispuestas en dos series subiguales, 2,2-3,8 x 0,5-1,1 mm, obovadas a ovadas, ápice redondeado y levemente mucronado, margen anchamente escarioso, enteras, tricomas glandulares presentes; receptáculo plano, alveolado; flores 16-40, corolas con tubo 0,6-1,9 mm de longitud, labio abaxial 1,53,4 x 0,7-1,9 mm, elíptico a ovado, labio adaxial 1,1-2 x 0,4-1 mm, ovado, blanco; anteras 1,4-2,1 $\mathrm{mm}$ de longitud, apéndice conectival $0,4-0,7 \mathrm{x}$ 0,1-0,3 mm, estilo 1,3-2,9 mm de longitud, ramas estilares 0,3-0,9 mm de longitud; cipselas 0,5-1,4 $\mathrm{mm}$ de longitud, obovoides.

Distribución y hábitat: Sur de Brasil, Uruguay y noreste de Argentina (Mondin \& Baptista, 1996; Katinas \& Crisci, 2008). En Uruguay hay registros de P. bupleurifolia, para los departamentos de Canelones, Cerro Largo, Durazno, Florida, Lavalleja, Maldonado, Montevideo, Paysandú, Salto, San José, Soriano y Tacuarembó (Fig. 4). Habita en praderas algo saladas (Cabrera 1953), en laderas y bajos fértiles, en campos bajos y arenosos, a orillas de lagunas, ríos, bañados y en prados uliginosos (Arechavaleta, 1906). Panphalea bupleurifolia puede desarrollarse en ambientes algo más secos (pero próximos a los anteriormente descriptos), en donde alcanza un menor desarrollo en altura.

Categoría de conservación: Preocupación menor (LC).

Fenología: Florece de octubre a marzo.

Especimenes examinados: URUGUAY. Dpto. Canelones: Santa Lucia, 1-XI-1869, Gibert 1392 (MVM); Ruta Interbalnearia km 27,5, 27-XI-1967, Lema s.n. (MVFA 6737). Dpto. Cerro Largo: Ruta 44 y Río Negro, S 3204'38,4"'W 5441'8”, 25-X2003, Bonifacino \& Sytsma 836 (MVFA); Bañados del Río Negro, entre arroyo Palleros, 17-XII-1937, Gallinal et al. 1703 (LP); Palleros y Acegua, campos del Dr. Alejandro Gallinal, 3-I-1936, Rosengurtt 1023 (LP); Escuela de Agronomía, bañado de Medina, alrededor de Tajamar, 20-X-1969, Olano et al. s.n. (LP, MVFA 8699); Ruta 7, entre Villa Viñoles y La Zotea, 21-X-1992, Izaguirre et al. s.n. (MVFA 21057). Dpto. Durazno: Villasboas, 14-XI-1900, Osten 4374 (MVM). Dpto. Florida: Ruta 6, km 198, 25-III-1994, Bayce et al. s.n. (MVFA 24679); Santa Clara, Cerro Colorado, 28IX-1926, Herter 19092 (MVM); Cerro Colorado, estancia San Pedro, 20-XII-1937, Gallinal et al. 2525 (LP); Paso Severino, XI-1942, Lombardo s.n. (MVJB 4109); Paso Severino, XI-1942, Lombardo s.n. (MVJB 6107); Cerro Colorado, X-1938, Otto de Mata 1133 (MVJB 2892); Santa Clara. Parcela 
Bol. Soc. Argent. Bot. 49 (1) 2014
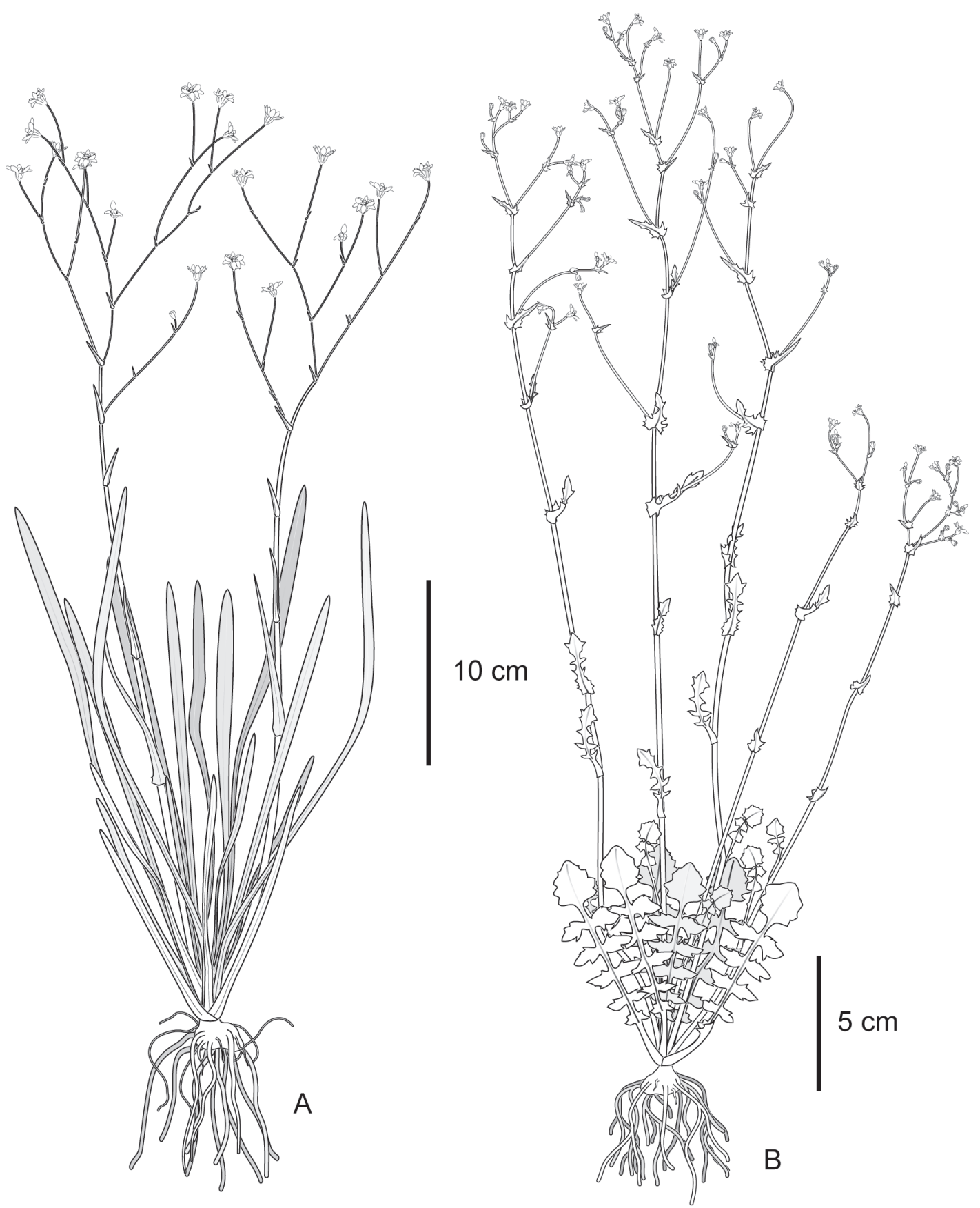

Fig. 2. Aspecto general de la planta. A: Panphalea bupleurifolia. B: Panphalea cardaminifolia. (A: Izaguirre s.n., MVFA 21057; B: Del Puerto \& Marchesi s.n., MVFA 11399).

de "Las Lincoln", 16-XI-1934, Rosengurtt 297 (MVFA); Cerro Colorado, estancia Elvira de E. Algorta Cancusso, 16-XII-1936, Rosengurtt 720 (MVJB 2159); Estancia San Pedro, Cerro
Colorado, XII-1937, Rosengurtt 720 (MVM 8191); Picada Castro, estancia Rincón de Santa Elena, arroyo Mansavillagra, 6-XI-1946, Rosengurtt 5749 (MVFA); Río Yí y arroyo Mansavillagra, 24-XII- 
1936, Rosengurtt 720 (LP). Dpto. Lavalleja: Ruta 8, arroyo Cebollatí, S 3349'51,6" W 5445'47,9”, 2-XI-2010, Bonifacino et al. 4055 (MVFA). Dpto. Maldonado: localidad no indicada, XI-1932, Lombardo s.n. (MVJB 7694). Dpto. Montevideo: Montevideo, XI-1867, Gibert 122, 1384, 1467 (MVM); XII-1874, Gibert s.n. (LP 5913); Carrasco, I-1936, Lombardo s.n. (MVJB 4769). Dpto. Paysandú: Arroyo Rabón y Ruta 3, S 32²7'19,9', W 57 57'53,9”, 28-X-2003, Bonifacino \& Sytsma 919 (MVFA); Campo de Daniel Durán, Ruta 26, próximo al arroyo Soto, 20-X-1996, Marchesi s.n. (MVFA 26212). Dpto. Salto: Cerca de orillas arroyo Guayabos y camino desde ruta 26, 2-XI1990, Bayce \& Marchesi s.n. (MVFA 19956). Dpto. San José: Arazatí, 24-XII-1939, Legrand 1695 (MVM); Arazatí, campo de fábrica Nac.Papel, 17XI-1978, Marchesi s.n. (MVFA 15813); Arazatí, campo Nro.14 FNP., 10-XI-1981, Marchesi s.n. (MVFA 16863); Arazatí, Arroyo Sauce, 6-XII1936, Rosengurtt 1716 (LP); Barra de Santa Lucía, 16-XI-1930, Osten 22061 (MVM); Arazatí, XI1933, Rosa Mato 669 (MVM 9860). Dpto. Soriano: Juan Jackson, estancia Monzón-Heber, X-1939, Rosengurtt et al. 4290 (MVFA). Dpto. Tacuarembó: Paso de los Toros, 5-XII-1900, Berro 608 (MVFA).

Panphalea cardaminifolia Less. Linnaea 5: 9. 1830. Tipo: Uruguay [Brasilia meridionali]. Montevideo, Sellow 1553 (Holotypus B, destruido, foto F 16095!). Uruguay. Montevideo, Sellow s.n. (Isotypus K, foto!). Fig. 2B.

Hierbas perennes, 20-45 cm de altura, con cortos rizomas; tallos erectos, a veces fistulosos, tricomas glandulares presentes; hojas inferiores 4,1-13,1 x 0,8-1,5 cm, pecíolo no definido, plano, envainador; lámina linear a obovada, lirado-pinnatisecta a pinnatipartida, ápice agudo, base atenuada, lóbulo apical ovado, lóbulos de margen entero a dentado, tricomas glandulares presentes; hojas superiores $0,7-8,3 \times 0,5-1,2 \mathrm{~cm}$, gradualmente reducidas en tamaño hacia el ápice, semiamplexicaules, similares a las inferiores a ovadas pinnatífidas, ápice agudo, base truncada, lóbulos de margen entero a dentado, tricomas glandulares presentes; capitulescencias paniculiformes, capítulos 3-36, pedicelos de 0,3$3,2 \mathrm{~cm}$ de longitud, tricomas glandulares presentes, abundantes; bractéolas 1-3, 0,9 x 0,5 mm; involucro
2,4 x 1,9 mm, cilíndrico; filarias 6-8, dispuestas en una serie, 1,5-3,3 x 0,5-1 mm, elípticas, ápice agudo a obtuso, a veces mucronado, margen escarioso, enteras a dentadas hacia el ápice, tricomas glandulares presentes, abundantes en el ápice; receptáculo plano, alveolado; flores 12-14, corolas con tubo 1,2-1,6 mm de longitud, labio abaxial 2,32,5 x 1-1,5 mm, ovado a oblongo, labio adaxial 1,21,4 x 0,6-1 mm, ovado, blanco; anteras 1,5-1,8 mm de longitud, apéndice conectival 0,5-0,7 x 0,2 mm; estilo 1,9-2,5 mm de longitud, ramas estilares $0,4-$ $0,5 \mathrm{~mm}$ de longitud; cipselas $0,6-1 \mathrm{~mm}$ de longitud, obovoides.

Distribución y hábitat: Sur de Brasil y Uruguay (Mondin \& Baptista, 1996; Katinas \& Crisci, 2008). En Uruguay existen dos registros de $P$. cardaminifolia, uno para el departamento de Artigas (Fig. 4) y el otro para Montevideo, correspondiéndose este último con el material tipo. De acuerdo con Arechavaleta (1906), la especie habita en terrenos húmedos, lugares bajos, bañados, cañadas, y campos graminosos.

Categoría de conservación: Datos insuficientes (DD).

Fenología: Florece en octubre, probablemente extendiéndose hasta febrero.

Especímenes examinados: URUGUAY. Dpto. Artigas: Ruta 3, km 625 y arroyo Itacumbú, 11-X1972, Del Puerto \& Marchesi s.n. (MVFA 11399).

Nota: El espécimen Del Puerto \& Marchesi s.n. (MVFA 11399) sería la única muestra de Panphalea cardaminifolia presente en herbarios uruguayos. No se pudo relocalizar a campo la población a partir de la cual se obtuviera el espécimen en cuestión. Este ejemplar posee lóbulos foliares algo más anchos que el holotipo de la especie en B, pero se asemeja al isotipo en K. Además, las hojas inferiores en el ejemplar MVFA 11399 son algo diferentes a las del holotipo, pues en este último poseen el lóbulo apical con cinco lóbulos muy conspicuos ( $v s$. menos conspicuos en MVFA 11399) y los laterales son hasta 2-3-dentados (vs. 4-dentados). Es posible que futuras colecciones corroboren la existencia de variación foliar en esta especie.

El ejemplar MVFA 11399 se asemeja a P. maxima 
por sus hojas muy recortadas con un gran lóbulo apical y por el tipo de inflorescencia. Sin embargo, este ejemplar se diferencia por su menor porte y por los lóbulos foliares laterales con incisiones mucho más profundas.

Panphalea commersonii Cass. Bull. Sci. Soc. Philom. Paris: 111. 1819. Tipo: Uruguay. Montevideo, Commerson s.n. (Holotypus P 670354, foto!; Isotypus P 670355, foto!). Fig. 3A.

Hierbas perennes, 6-35 $\mathrm{cm}$ de altura, con base tuberosa o xilopodio; tallos erectos, estriados, tricomas glandulares presentes, escasos; hojas inferiores 1,7-17,3 x 0,7-3,7 cm, pecíolo definido, cilíndrico, 1-14,5 cm de longitud; lámina 0,7-2,8 x 0,7-3,7 cm, anchamente ovada a orbicular, entera a 3-5-lobulada, base cordada, ápice obtuso a levemente acuminado, margen apenas dentado, tricomas glandulares presentes, escasos; hojas superiores 0,34 × 0,1-0,85 cm, gradualmente reducidas en tamaño hacia el ápice, semiamplexicaules, ovadas, elípticas, largamente atenuadas hacia ápice, ápice agudo, base a veces dentada, margen entero, tricomas glandulares presentes, escasos; capitulescencias corimbiformes, capítulos 2-26, pedicelos 0,1-5,5 $\mathrm{cm}$ de longitud, tricomas glandulares presentes; bractéolas 1-4, 1-2,8 x 0,5-1 mm, ovadas; involucro 3,5-5,1 x 2-3 mm, cilíndrico; filarias 5-8, dispuestas en una serie, 2,7-4,9 × 0,6-1,3 mm, elípticas a obovadas, ápice hendido y mucronado, margen anchamente escarioso, enteras, tricomas glandulares presentes; receptáculo plano o convexo, levemente alveolado; flores 6-21, corolas con tubo 1,1-2 mm de longitud, labio abaxial 1,9-3,7 x 1,1-2 mm, ovado a elíptico, labio adaxial 1,3-2,3 x 0,5-1,2 mm, ovado, blanco; anteras 1,5-2,1 mm de longitud, apéndice conectival 0,6-0,9 x 0,1-0,3 mm; estilo 1,7-3,9 mm de longitud, ramas estilares $0,4-0,9 \mathrm{~mm}$ de longitud; cipselas $0,6-1,5 \mathrm{~mm}$ de longitud, obovoides.

Distribución y hábitat: Sur de Brasil y Uruguay (Mondin \& Baptista, 1996; Katinas \& Crisci, 2008). En Uruguay hay registros de P. commersonii para los departamentos de Canelones, Cerro Largo, Florida, Maldonado, Montevideo, Paysandú, Rivera, Salto y Soriano (Fig. 4). Habita en campos abiertos, graminosos, cerros y sierras (Arechavaleta, 1906), cerca de la base y en laderas pedregosas.
Categoría de conservación: Preocupación menor (LC).

Fenología: Florece de agosto a abril.

Especímenes examinados: URUGUAY. Dpto. Canelones: Arroyo Sarandí, 5-IX-1961, Izaguirre 203 (MVFA). Dpto. Cerro Largo: Escuela de Agronomía, bañado de Medina, 6-X-1961, Del Puerto 173 (MVFA); Palleros, Cuchilla Negra Norte, 5-XII-1937, Rosengurtt 1560 (MVFA). Dpto. Florida: Cerro Colorado, Santa Elvira, Parcela 1, XII-1937, Rosengurtt 1996 (LP, MVFA); Sauce, San Pedro, 30-XI-1938, Rosengurtt 3299 (MVFA). Dpto. Maldonado: Pan de Azúcar, III1899, Arechavaleta s.n. (MVM); Cerro Aigúa al sur, 20-XI-1965, Rosengurtt 9846 (MVFA). Dpto. Montevideo: X-1876, Arechavaleta s.n. (MVM); Cerro, 25-IV-1983, Gago et al. s.n. (MVJB 23236); Cuchilla Juanico, X-1863, Gibert s.n. (MVM); Cerro, XI-1866, Gibert s.n. (MVM); Cerro, 13-X1971, Izaguirre et al. s.n. (MVFA 10739); 12-IX1940, Legrand 2248 (MVM); Campo del barrio "La Floresta", VIII-1926, Lombardo s.n. (MVJB 3505); Atahualpa, X-1926, Lombardo s.n. (MVJB 788); Cerro, X-1937, Lombardo s.n. (MVJB 3149); Cerro, IX-1926, Marchesi s.n. (MVJB 4994); 1926, Marchesi s.n. (MVJB 4995); Punta Yeguas, 8-X-1932, Osten 22676 (MVM); Parque Lecocq, 20-IX-1949, Rosengurtt 5720 (MVFA); Cerro de Montevideo, 18-X-1983, Scarlato et al. s.n. (MVJB 22946); Alrededores de Montevideo, sin fecha, Chebataroff 232 (LP). Dpto. Paysandú: Campo El Refugio. Pandule, S 32 $22^{\prime} 41^{\prime \prime}$ W 57 $25^{\circ} 54^{\prime \prime}$, 17-X-1995, Marchesi \& Vignale s.n. (MVFA 24923); Arroyos Guaviyú y Sarandí, 1/3-IX-1948, Rosengurtt 5184 (MVFA). Dpto. Rivera: Gajo del Lunarejo, 1-XI-2003, Bonifacino \& Langström 935 (MVFA). Dpto. Salto: Rincón de Guayaca, 5-IX1900, Osten 3616 (MVM). Dpto. Soriano: Vera, 23-X-1899, Berro 683 (MVFA).

Panphalea heterophylla Less. Linnaea 5: 8. 1830. Tipo: Uruguay [Brasiliae meridionalis]. Montevideo, Sellow 1328 (Holotypus B, destruido, foto F 16096!). Brasil. Brasilia, Sellow 4048 (Isotypus K, foto!), Sellow s.n. (Isotypus P, foto!), Rio Grande, Sellow s.n. (Isotypus P, foto!). Fig. 3B. Pamphalea tweedii Gardn. \& Field. Sertum 


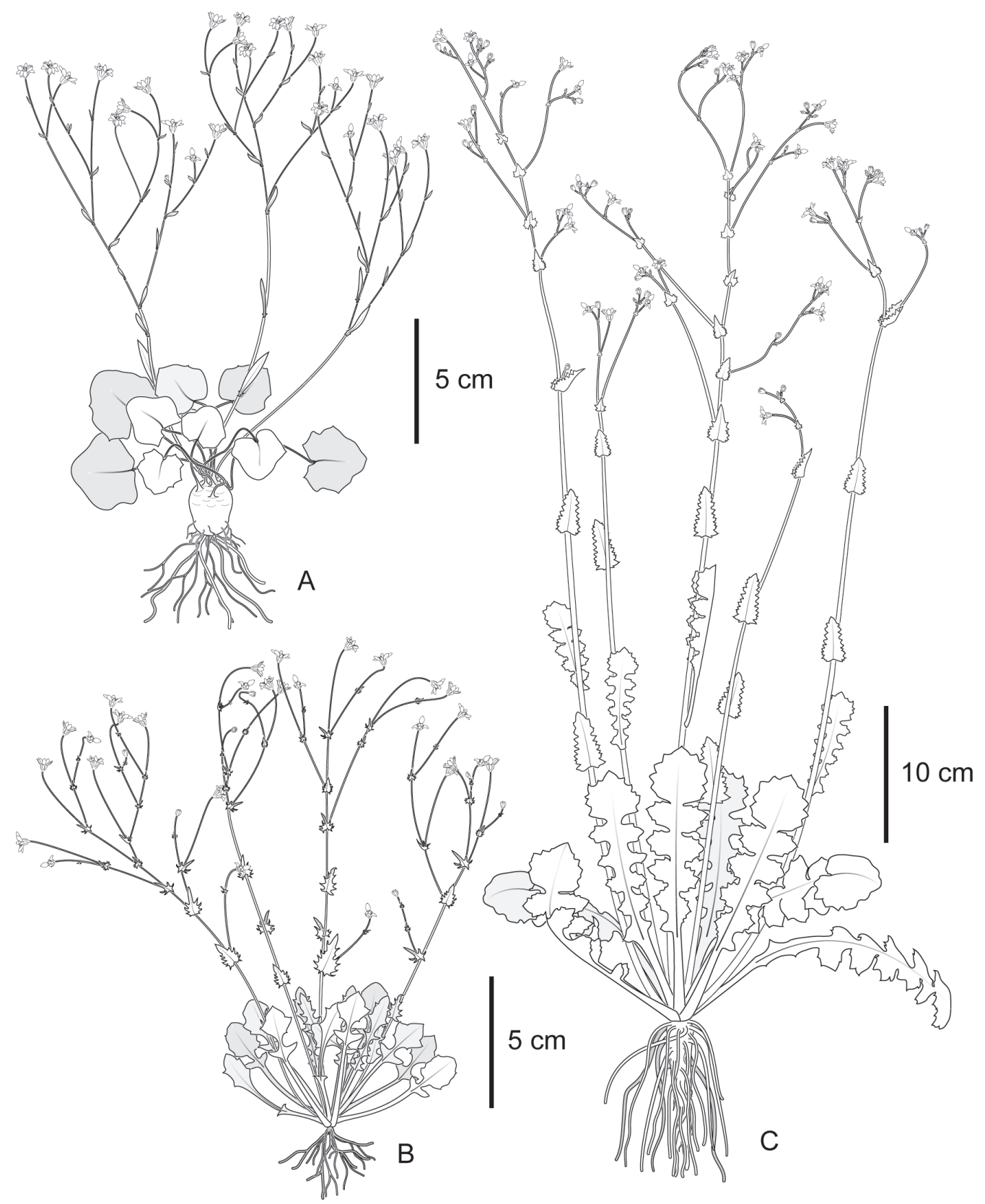

Fig. 3. Aspecto general de la planta. A: Panphalea commersonii. B: Panphalea heterophylla. C: Panphalea maxima. (A: Berro 683, MVFA; B: Bonifacino \& Sytsma 833, MVFA; C: Bonifacino et al. 4052, MVFA).

Plantarum 1: t.21, 1844. Tipo: Uruguay. Salto del Uruguay, 1837, Tweedie s.n. (Holotypus K, foto!).

Hierbas anuales, delicadas, 2,7-20(-23) cm de altura, con varios tallos ascendentes; tallos erectos, tricomas glandulares presentes, escasos; hojas inferiores $0,45-8,4 \times 0,14-1,3 \mathrm{~cm}$, pecíolo no definido, plano, envainador; lámina orbicular, ovada a elíptica, lirado-pinnatífida, base asimétrica o simétrica, ápice obtuso a agudo, a veces acuminado, 
margen entero o dentado, tricomas glandulares presentes, escasos; hojas superiores 0,3-2,8 x 0,1-0,8 cm, gradualmente reducidas en tamaño hacia el ápice, semiamplexicaules, similares a las inferiores a ovadas, atenuadas hacia el ápice, ápice agudo a acuminado, base truncada, margen entero, generalmente dentado a lobado hacia la base, lóbulos de ápice agudo, tricomas glandulares presentes, escasos; capitulescencias corimbiformes, a veces capítulos solitarios en las plantas pigmeas, capítulos $1-20$, pedicelo $0,1-8,4 \mathrm{~cm}$ de longitud, tricomas glandulares presentes; bractéolas 1-3, 0,51,9 × 0,4-1 mm, ovadas; involucro 2,5-3,7 x 1,9$2,9 \mathrm{~mm}$, cilíndrico; filarias 5-8, dispuestas en una serie, 1,5-3,4 x 0,6-1,5 mm, elípticas a obovadas, a veces oblongas, ápice hendido y mucronadoespinoso, margen anchamente escarioso, enteras, tricomas glandulares presentes; receptáculo plano, tuberculado; flores 11-23, corolas con tubo 1-1,9 mm de longitud, labio abaxial 1,8-3,2 x 0,8-2 mm, elíptico a ovado, labio adaxial 1-1,9 x 0,5-1,3 mm, ovado, amarillo; anteras 1,1-1,8 $\mathrm{mm}$ de longitud, apéndice conectival $0,4-0,7 \times 0,1-0,3 \mathrm{~mm}$, elíptico a ovado; estilo 1,4-2,4 $\mathrm{mm}$ de longitud, ramas estilares 0,3-0,6 mm de longitud; cipselas 0,6-1,5 $\mathrm{mm}$ de longitud, obovoides a elipsoides.

Distribución y hábitat: Sur de Brasil, Uruguay y noreste de Argentina (Mondin \& Baptista, 1996; Katinas \& Crisci, 2008). En Uruguay hay registros de $P$. heterophylla para los departamentos de Artigas, Canelones, Cerro Largo, Colonia, Durazno, Flores, Florida, Lavalleja, Maldonado, Montevideo, Paysandú, Río Negro, Rivera, Rocha, Salto, San José, Soriano, Tacuarembó y Treinta y Tres (Fig. 4). Habita en campos graminosos, pedregosos, cerros y sierras (Lombardo, 1983), campos arenosos, bajos, algarrobal sin pastoreo, y bañados. Bajo sombra se encontraron ejemplares de mayor altura. (LC).

Categoría de conservación: Preocupación menor

Fenología: Florece de septiembre a diciembre.

Especimenes examinados: URUGUAY. Dpto. Artigas: Ruta 3, km 608, 11-X-1972, Del Puerto \& Marchesi s.n. (LP, MVFA 11338); Puerto Bentún, sobre río Cuareim, próximo a Bella Unión, 11X-1972, Del Puerto \& Marchesi s.n. (MVFA
11383). Dpto. Canelones: Paso de Pache, XI-1925, Lombardo s.n. (MVJB 1180); Paso de Pache, XI-1929, Lombardo s.n. (MVJB 2632); Santa Lucia, parcela del 1, 12-XI-1934, Rosengurtt 127 (MVFA); Santa Lucia, parcela del 2, 12-XI-1934, Rosengurtt 147 (MVFA); Paso Cuello, Parcela 1, 12-XI-1934, Gallinal 127 (LP). Dpto. Cerro Largo: Ruta 44 y Río Negro, S 3204'38,4” W 54\%41'8", 25-X-2003, Bonifacino \& Sytsma 833 (MVFA); Arroyo Cordobés y arroyo Pablo Páez, 4-X-1961, Rosengurtt et al. 128 (MVFA). Dpto. Colonia: Molino Quemado, 12-X-1957, Arrillaga 688 (MVFA); Carmelo, 6-XII-1934, Cabrera 3201 (LP, MVM); Riachuelo, X-1949, Fabris \& Gebhard 5 (LP); Riachuelo, 1-XI-1960, Cabrera 13625 (LP); Riachuelo, 11-X-1936, Cabrera 3817 (LP). Dpto. Durazno: Estancia La Paz, arroyo Cordobés, 3-X-1961, Del Puerto s.n. (MVFA 67); Molles, 30-IX-1899, Osten 3815 (MVM); Ruta 5 al N del arroyo Villasboas. Campos de Castells, 2-XI-1973, Rosengurtt 11497 (MVFA). Dpto. Flores: Porongos, 3-IX-1899, Osten 3777 (MVM); Río Yí entre arroyo Matanzas y Carpintería, estancia de Quinteros, 25-XI-1936, Rosengurtt 508 (LP, MVFA); Arroyo Grande, estancia Santa Adelaida, parcela 25, 17-XI1937, Gallinal et al. 997 (LP); 25-XI-1937, Gallinal et al. 1143 (LP). Dpto. Florida: Casupá, 18-XI-1962, Del Puerto s.n. (MVFA 6872); Cerro Colorado, estancia San Pedro, Sauce, XII-1937, Gallinal et al. PE-3255 (LP); Cerro Colorado, estancia San Pedro, parcela Bichadero, 14-XII-1936, PE-207 (LP); Florida-Timote- Santa Clara, 19-XI-1934, Gallinal 41 (LP); Santa Clara, cerro Colorado, 28IX-1926, Herter s.n. (MVM); Campos de Gala mala, X-1925, Lombardo s.n. (MVJB 581); Río Santa Lucía, pueblo 25 de Agosto, 21-XI-1935, Rosengurtt 1097 (LP); Estancia San Pedro, cerro Colorado, XII-1936, Gallinal et al. PE-207 (MVM 8192). Dpto. Lavalleja: Ruta 40, sierra de Polanco, 24-IX1993, Bayce et al. s.n. (MVFA 22283); Cerro de los Cuervos, 17/18-X-1998, Bonifacino \& Speroni s.n. (MVFA 28754); Cerros del Molino, Sierra de Minas, XII-1939, Chebataroff 3047 (LP); Sierras de Minas, Penitente, 5-XI-1933, de Medina 148, 1545 (MVM); Cerro Arequita, 24-XI-1963, Marchesi 747 (MVFA); Minas, cerro Arequita, 3-X-1937, Rosengurtt 1354 (MVFA). Dpto. Maldonado: Parque Lussich, 12-X1956, Arrillaga 424 (MVFA); Cerro de las Animas, 29-X-2003, Bonifacino \& Langström 928 (MVFA); Punta Ballena, X-1937, Lombardo s.n. (MVJB 


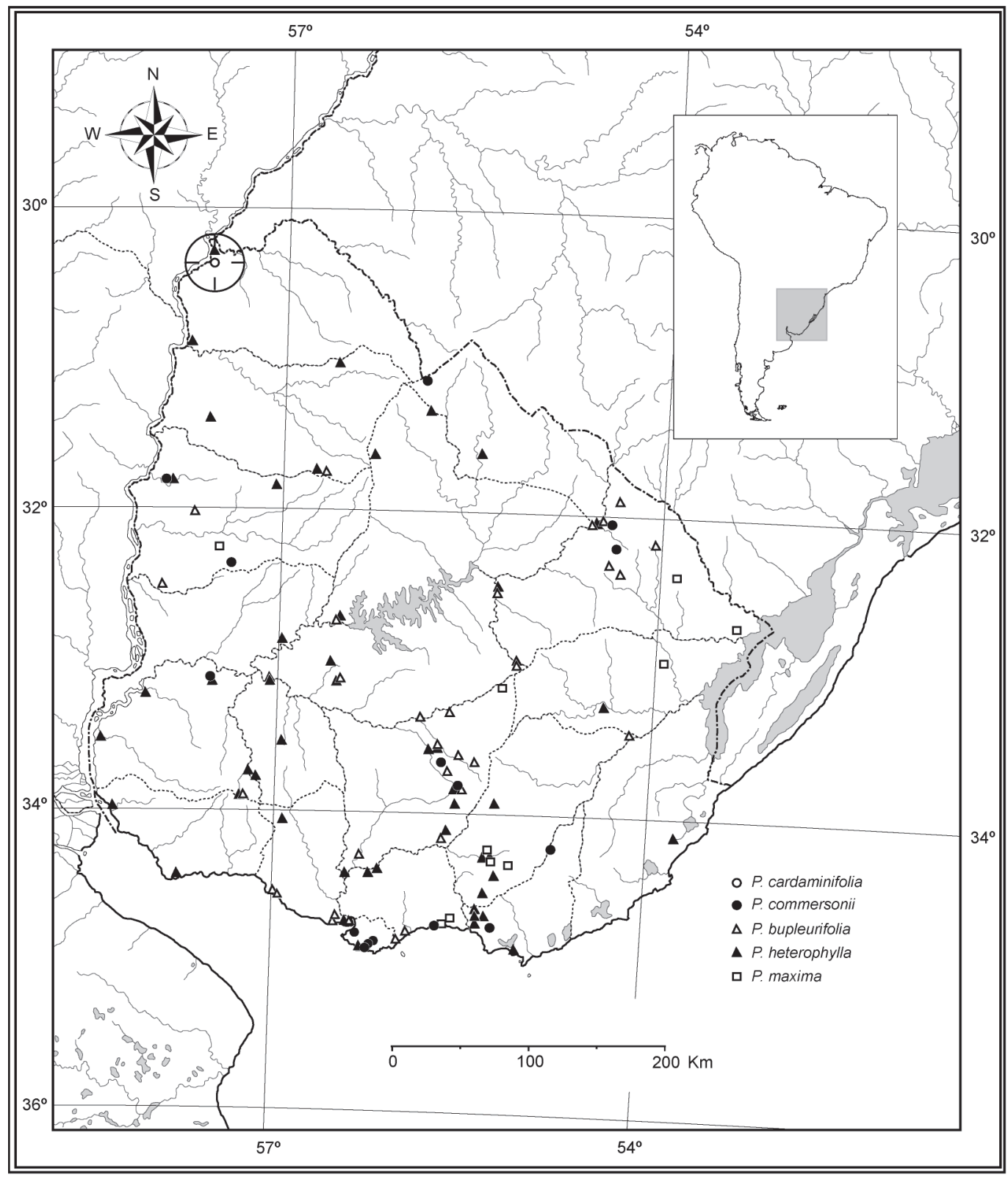

Fig. 4. Mapa de distribución geográfica de las especies de Panphalea en Uruguay.

3148); Cerro Tupambaé, 6-X-1963, Marchesi 471 (MVFA); Sierras Animas, 5-XI-1931, Osten 22344 (MVM). Dpto. Montevideo: X-1877, Arechavaleta s.n. (MVM); XI-1880, Gibert s.n. (MVM); X-1867, Gibert s.n. (LP); 3 XI 1907, Osten s.n. (MVM). Dpto. Paysandu: Ruta 26, km 124, 16-XI-1977, Marchesi \& Ferrés s.n. (MVFA 14342); Arroyo Guaviyú y arroyo Sarandí, 1/2-IX-1948, Rosengurtt 5183 (MVFA). Dpto. Río Negro: Balsa al norte, 6-XI1913, Berro 7012 (MVFA); Palmares de Porrúa, 16/19-X-1995, Brescia et al. s.n. (MVFA 26801). Dpto. Rivera: Mandiyú, S 30³0,6' W 57²41,1', 15-X-2004, Brussa s.n. (MVJB 24564); El arenal, 9-XII-2003, Delfino et al. s.n. (MVJB 24826); 
Minas de Corrales, arroyo Corrales, S 31'35'62,5" W 55'29'9", 7-XI-2005, Delfino et al. s.n. (MVJB 23372). Dpto. Rocha: Sierra de los Difuntos, Colonia Don Bosco, S 34 5'7' W 5346'18', 18-IX-2006, Bonifacino 2455 (MVFA). Dpto. Salto: Estancia Los Venados. Puntas del Tapado, 16-XI-1995, Bonifacino s.n. (MVFA 25119); Ruta 31, entre San Antonio y Valentín, 21-IX-1970, Del Puerto et al. s.n. (MVFA 9328); Camino a Guaviyú próximo a ruta 4, 31-VIII-1969, Del Puerto \& Marchesi s.n. (MVFA 8585). Dpto. San José: Sierra Mahoma, 26-IX-1962, Del Puerto 2170 (MVFA); 14 X 1930, Osten 22005 (MVM). Dpto. Soriano: Vera, 12-IX-1898, Berro 684 (MVFA); Campo Battro, 24-XII-1913, Berro 7056 (MVFA); Mercedes, 27X-1914, Berro 7708 (MVFA); Mercedes, Mauá, X-1891, Osten 3071 (MVM); Juan Jackson, estancia Monzón-Heber, XI-1937, Gallinal et al. 768 (LP); Santa Elena, parcela del 21, 5-XI-1934, Gallinal 61 (LP); Arroyo San Salvador, 24-IX-1873, Berg 25 (LP). Dpto. Tacuarembó: Cañada del Tala y ruta 31 , S 31 38'34,6" W 56 $18^{\circ} 3,2^{\prime}$ ", 27-X-2003, Bonifacino \& Sytsma 879 (MVFA); Ruta 5, Km.257, a un lado de la carretera, 19-IX-1986, Izaguirre et al. s.n. (MVFA 18436). Dpto. Treinta y Tres: Río Olimar a la altura de Treinta y Tres, 22-X-1969, Olano et al. s.n. (MVFA 8804).

Panphalea maxima Less. Linnaea 5: 9. 1830. Tipo: Uruguay [Brasilia meridionalis]. Montevideo, Sellow 1365 (Holotypus B, destruido, foto $\mathrm{F}$ 16097!). Neotypus aquí designado: Uruguay.

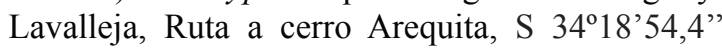
W 5515'17,6", 2-XI-2010, Bonifacino et al. 4052 (MVFA!). Duplicado del neotypus: LP! Fig. 3C.

Hierbas perennes, generalmente robustas, 9-80 $\mathrm{cm}$ de altura, con rizoma desarrollado; tallos erectos, ocasionalmente fistulosos, estriados, tricomas glandulares presentes; hojas inferiores 5-20 x 1-4 cm, pecíolo no definido, plano, envainador; lámina linear a obovada, lirado-pinnatífida, base atenuada, a veces lóbulos inferiores desfasados, ápice obtuso a agudo, lóbulo apical ovado, lóbulos de margen crenado-dentado, con ápice espinoso-mucronado, enteros, tricomas glandulares presentes; hojas superiores 0,4-13 x 0,1-3,2 cm, gradualmente reducidas en tamaño hacia el ápice, semiamplexicaules, similares a las inferiores a ovadas pinnatífidas, base truncada, margen lobado, crenado-dentado, tricomas glandulares presentes; capitulescencias paniculiformes, capítulos 6-120, pedicelos 0,1-4,7 cm de longitud, tricomas glandulares presentes, abundantes; bractéolas 1-3, 0,6-2 x 0,3-0,9 mm, ovadas; involucro 2,9-3,6 x 2,1-2,9 mm, cilíndrico; filarias 6-12, dispuestas en una o dos series subiguales, 2,5-3,5 x 0,6-1,3 mm, elípticas a obovadas, ápice mucronado, margen escarioso, dentadas en general hacia el ápice, tricomas glandulares presentes, abundantes en el ápice; receptáculo plano, tuberculado; flores 8-24, corolas con tubo 1,5-2,2 $\mathrm{mm}$ de longitud, labio abaxial 2,1-3,7 x 1,3-2,1 mm, elíptico a ovado, labio adaxial 1,1-2 x 0,6-1,3 mm, ovado, blanco, amarillo en zona cercana al tubo; anteras 1,5-2,1 $\mathrm{mm}$ de longitud, apéndice conectival 0,5-1 x 0,1$0,3 \mathrm{~mm}$; estilo 2,2-3,9 $\mathrm{mm}$ de longitud, ramas estilares $0,4-0,8 \mathrm{~mm}$ de longitud; cipselas $0,6-1,6$ $\mathrm{mm}$ de longitud, obovoides.

Distribución y hábitat: Sur de Brasil y Uruguay (Mondin \& Baptista, 1996; Katinas \& Crisci, 2008). En Uruguay hay registros de P. maxima para los departamentos de Canelones, Cerro Largo, Florida, Lavalleja, Paysandú y Treinta y Tres (Fig. 4). Se encuentra en parajes húmedos, a veces sombríos (Arechavaleta, 1906), en sitios anegados con o sin pastoreo, y a los lados de éstos, donde alcanzan menores alturas; con ejemplares de Juncus spp., Cyperus spp., Carex spp., Rhynchospora spp., Hydrocotyle spp., Ludwigia peploides (Kunth) P.H. Raven, especies de la familia Poaceae, Sisyrinchium spp., Plantago spp., Senecio bonariensis Hook. \& Arn., Baccharis spp. y Eclipta spp.

Categoría de conservación: Preocupación menor (LC).

Fenología: Florece de octubre a febrero.

Especimenes examinados: URUGUAY. Dpto. Canelones: Sierras de Afilar, 2-XI-1928, Osten 20099 (MVM); Arenales entre arroyo Sarandí y la Floresta, 22-II-1936, Rosengurtt 147 (MVFA). Dpto. Cerro Largo: Arroyo Malo, ruta 26, 22-XI1968, Lema et al. s.n. (MVFA 7890); Cañada de los Ceibos, cerca de arroyo Tacuarí, S 3242'30,3" W 5322', 23-X-2004, Marchesi et al. s.n. (MVFA). Dpto. Florida: Cerca de arroyo Valentines, S 
3314'8,9"' W 55¹4'51', 18-XI-2009, Marchesi 3031 (MVFA). Dpto. Lavalleja: Ruta a cerro Arequita, S $34^{\circ} 18^{\prime} 54,4^{\prime \prime}$ W 55 $5^{\circ} 15^{\prime} 17,6$ ", 2-XI2010, Bonifacino et al. 4052 (MVFA); Río Santa Lucia y ruta a cerro Arequita, 1-XII-1979, Marchesi s.n. (MVFA 16440); Ruta 8, km 139, 1-XII-1979, Marchesi s.n. (MVFA 16463). Dpto. Paysandú: Cueva del Tigre, puntas del arroyo Nacurutú, I-1942, Chebataroff 7001 (LP). Dpto. Treinta y Tres: $1 \mathrm{~km}$ de arroyo Parao, cerca de Vergara, 21-X1969, Olano et al. s.n. (MVFA 8768).

\section{Agradecimientos}

Deseamos agradecer a los curadores de los herbarios MVFA, MVM, MVJBy LP por permitirnos el acceso a las colecciones. MB agradece a Facultad de Agronomía de UDELAR (Montevideo) y a PEDECIBA por el apoyo financiero para realizar el trabajo de campo.

\section{Bibliografía}

ARECHAVALETA, J. 1906. Flora Uruguaya. Anales Mus. Nac. Montevideo 6: 447-452.

CABRERA, A. L. 1953. Las especies del género Pamphalea (Compositae). Notas Mus. La Plata, Bot. 16: 225-237.

CABRERA, A. L. 1959. Compositae catarinenses novae. Bol. Soc. Argent. Bot. 7: 188-200.

CRISCI, J. V. 1974. A numerical-taxonomic study of the subtribe Nassauviinae (Compositae, Mutisieae). $J$. Arnold Arbor. 55: 568-610.

DE CANDOLLE, M. 1812. Observations sur les plantes Composées, ou Syngenèses, troisième mémoire: Sur les Composées a corolles labiées ou Labiatiflores. Ann. Mus. Natl. Hist. Nat. 19: 59-72.

GIBERT, E. 1873. Enumeratio Plantarum Sponte Nascentium Agro Montevidensi. Sumptibus Societatis La Asociación Rural del Uruguay, Montevideo.

HERTER, G. 1930. Estudios Botánicos en la Región Uruguaya. IV. Florula Uruguayensis, plantae vasculars. Sumptibus rei publicae, Montevideo.

IUCN. 2012. IUCN Red List Categories and Criteria: Version 3.1. Second edition. Gland, Switzerland and Cambridge, UK: IUCN. iv + 32pp.
IUCN Standards and Petitions Subcommittee. 2013. Guidelines for Using the IUCN Red List Categories and Criteria. Version 10.1. Standards and Petitions Subcommittee. Disponible en: http://www. iucnredlist.org/documents/RedListGuidelines.pdf.

KATINAS, L. 1995. Panphalea Lag. En: HUNZIKER, A. T. (ed.), Fl. Fanerog. Argentina 13: 36-37.

KATINAS, L. 2008. Panphalea Lag. En: ZULOAGA, F. O., O. MORRONE \& M. J. BELGRANO (eds.), Catálogo de las plantas vasculares del Cono Sur (Argentina, sur de Brasil, Chile, Paraguay y Uruguay). Monogr. Syst. Bot. Missouri Bot. Gard. 107: 1420-1421.

KATINAS, L. \& J. V. CRISCI. 2008. Reconstructing the biogeographical history of two plant genera with different dispersion capabilities. J. Biogeogr. 35: 1374-1384.

KATINAS, L., J. PRUSKI, G. SANCHO \& M. C. TELLERÍA. 2008. The subfamily Mutisioideae (Asteraceae). Bot. Rev. 74: 469-716.

LAGASCA, M. 1811. Disertación sobre un nuevo orden de plantas de la clase de las Compuestas. Amen. Nat. Españ. 1: 26-44.

LOMBARDO, A. 1983. Pamphalea Lag. Fl. Montevidensis 2: 310-312.

MONDIN, C. A. \& L. R. BAPTISTA. 1996. Relacões biogeográficas da tribo Mutisieae Cass. (Asteraceae), sensu Cabrera, no Rio Grande do Sul. Comun. Mus. Ci. Tecnol. P.U.C.R.S., Ser. Bot. 2: 49-152.

MONGE, M. 2010. Panphalea Lag. En: FORZZA, R. C., J. F. A. BAUMGRATZ, C. E. M. BICUDO, A. A. CARVALHO JR., A. COSTA, D. P. COSTA, M. HOPKINS, P. M. LEITMAN, L. G. LOHMANN, L. C. MAIA, G. MARTINELLI, M. MENEZES, M. P. MORIM, M. A. N. COELHO, A. L. PEIXOTO, J. R. PIRANI, J. PRADO, L. P. QUEIROZ, V. C. SOUZA, J. R. STEHMANN, L. S. SYLVESTRE, B. M. T. WALTER \& D. ZAPPI (eds.), Catálogo de plantas e fungos do Brasil 1: 729. Jardim Botânico do Rio de Janeiro.

RAMAYYA, N. 1962. Studies on the trichomes of some Compositae I. General structure. Bull. Bot. Surv. India 4: 177-188.

UICN. 2012. Directrices para el uso de los Criterios de la Lista Roja de la UICN a nivel regional y nacional: Versión 4.0. Gland, Suiza y Cambridge, Reino Unido: UICN. iii + 43pp.

Recibido el 9 de mayo de 2013, aceptado el 8 de noviembre de 2013. 
